\section{Fluorescence of Solid Anthracene}

THE fluorescence of solid anthracene in light of wave-length $3650 \mathrm{~A}$. is changed from blue to green when very small quantities of naphthacene are present in solid solution. This is explicable by an 'exciton' mechanism whereby energy is handed on by resonance from molecule to molecule ${ }^{1}$. The graphs shown here represent a quantitative examination of the effect, the blue and green components of the fluorescence being measured by a photo-cell through Ilford filters 601 and 404 respectively. The upper graph shows for two temperatures the fall of the blue anthracene fluorescence and the rise of the green naphthacene fluorescence with concentration of the latter, expressed as quantum efficiencies with a probable error of 15 per cent. The emissions are about equal at a naphthacene concentration of $3 \times 10^{-5}$, and the green reaches a maximum at about $10^{-3}$. Above this concentration the colour of the emission changes to yellow as for pure naphthacene, and the efficiency decreases, evidently due to the overstepping of the limits of solid solution.

The supposed 'exciton' effect cannot be due to direct light absorption by the naphthacene owing to its feeble absorption at $3650 \mathrm{~A}$. To eliminate the possibility that re-absorption of blue anthracene fluorescence followed by re-emission as naphthacene fluorescence is the cause, solutions of anthracene and naphthacene in acetone were poured into aqueous gelatine solutions to produce milk-like sols the fluorescence of which could be measured under conditions of feeble light absorption. The lower graph shows as dotted curves the blue and green intensities for anthracene - naphthacene sols precipitated from one solution. These do not differ greatly from the curves of the upper graph for solid solutions made by

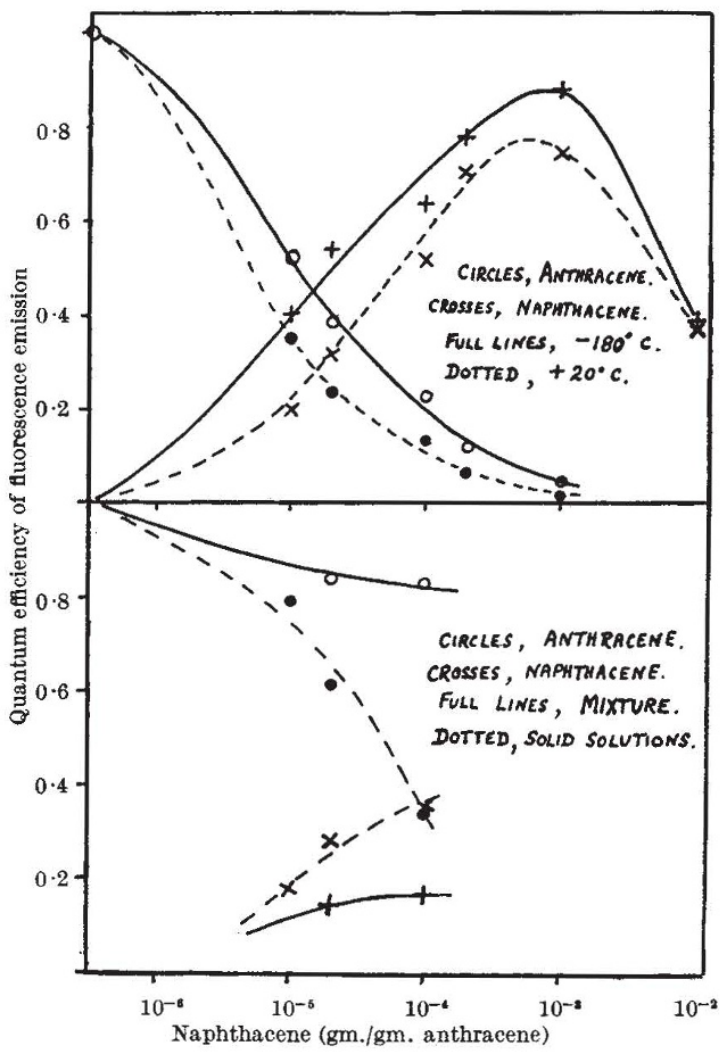

fusion. The full lines, however, relate to anthracenenaphthacene concentrations made by mixing separately prepared sols of pure anthracene and of anthracene-naphthacene mixtures of concentration $10^{-3}$. The differences for homogeneous and nonhomogeneous mixtures of similar composition are strong evidence in favour of the 'exciton' hypothesis.

E. J. BOWEN

E. MIKIEWICZ

Department of Physical Chemistry, Oxford.

${ }^{1}$ Bowen, E. J., Nature, 153, 653 (1943); J. Chem. Phys., 13, 306 (1945).

\section{Tetravalent Manganese as an Activator in Luminescence}

THE fluorescence of luminophors activated with manganese, and also of pure manganese compounds, is generally attributed to divalent manganese ${ }^{1}$. The spectra emitted vary between green and red, and the width of the bands also varies from case to case, both probably being dependent on the magnitude and the symmetry of the electric lattice field at the position in the lattice at which the manganese ion is placed.

There is no doubt that this explanation is correct for the greater part of the manganese fluorescence known, and the colour differences between the green and red fluorescence respectively of zinc and magnesium silicates activated with manganese are certainly not due to differences in the valency of the manganese.

We have found some systems, however, in which also manganese of a higher valency can cause fluorescence. The emission due to these ions is situated in the red and shows a fine structure which is unusually sharp for manganese phosphors. In these systems, namely, zinc aluminate, magnesium aluminate, $\alpha$-and $\beta$-aluminium oxide and magnesium titanate, all activated with manganese, the luminescence is markedly different according as the products are prepared under oxidizing or reducing conditions. The reduced products (except the titanate) show a green cathodo-luminescence. This emission is due to divalent manganese. The oxidized products show a deep red luminescence upon excitation by cathode rays or long-wave ultra-violet $(3650 \mathrm{~A} \text {. })^{2}$. This red fluorescence is due to manganese of a higher valency. In magnesium titanate the valency could be determined by chemical methods (titration), and was found to be between 3 and 4. That it is tetravalent manganese which is responsible for the red emission can further be proved by comparing the spectral distribution of the fluorescence of the salt when activated with manganese and with trivalent chromium respectively ${ }^{3}$.

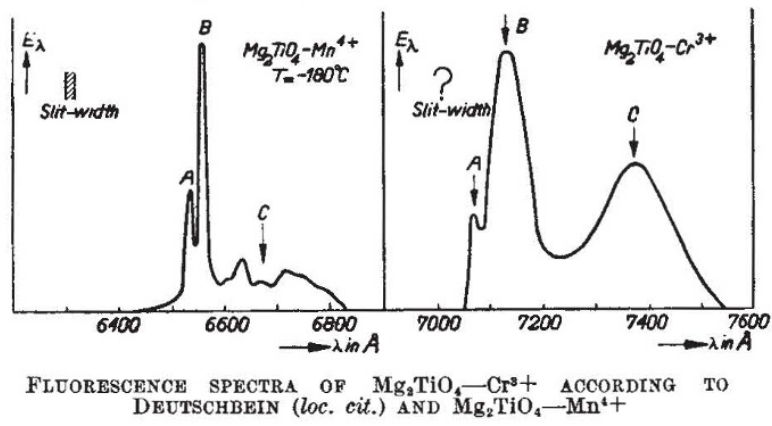

